MATEC Web of Conferences 22,03012 (2015)

DOI: $10.1051 /$ matec conf/ 20152203012

(C) Owned by the authors, published by EDP Sciences, 2015

\title{
Research on Grinding and Polishing Force Control of Compliant Flange
}

\author{
Chuang Li, Zhenhua Wang, Cheng Fan, Guodong Chen \& Ting Huang* \\ School of Mechanical and Electric Engineering, Soochow University, Suzhou, Jiangsu, China
}

\begin{abstract}
The automation of the grinding and polishing process is important to improve the production efficiency of the part surfaces. In this paper, a new compliant flange mounted on the end of the industrial robots for the robotic grinding and polishing force control is developed. With regard to the non-linear and time-varying problem of the contact force, the mathematical model of the new force control system was presented and the fuzzy PID control strategy was used to drive the proposed system. Especially, the air spring and electric proportional valve is studied to establish the model. The simulation results show that the selected control strategy has quick response and good robustness, which satisfies the real-time requirements of the grinding and polishing force control in processing.
\end{abstract}

Keywords: compliance; grinding and polishing force; air spring; fuzzy PID control

\section{INTRODUCTION}

Robots have provided an effective and economic method to achieve the industrial automation. Nowadays, they are widely applied to the automation of the industrial grinding and polishing process. Controlling the contact force is required for the industrial robots in many applications of precision operation. Therefore, the compliance based on force feedback is essential for the industrial robots $[1,2]$.

There are mainly two accepted methods of compliance controlling of industrial robots [3]. The first method is involved in applying force by the position of all the robot axes in unison. The second method uses the robot for positioning motion only, and an auxiliary compliant end-of-arm tool is added to supply a controlled force. The limitation of the first method is that it requires high location precision of the robots. In the practical engineering applications, it can decouple the force control and the position control by bringing in a compliant end-of-arm tool which is independent of the robots. For that method, the control strategy is easy and the response time delay of the coupling of the force/position can be avoided.

In this paper, we developed a new compliant flange for the robotic force control, which takes the air spring as actuator. The pneumatic actuator is applied in most compliant flange today by air cylinder or air spring and controls the grinding and polishing force through regulating the air pressure inside the cylinder or air spring. The advantages of the proposed methods include natural flexibility, compressibility, cleanness, easier collection and so on. Through adopting closed-loop control, the constant pressure regulation can be realized. The contact force can be adjusted by the input pressure. As a result, the flange can be used to regulate the contact force. We can use it to grind and polish in a pre-setting force and the processing quality of parts can be highly improved $[4,5]$.

This paper develops a compliant flange device mounted on the end of industrial robot for the robotic grinding and polishing process. The mathematical model of the grinding and polishing force control system is established. The simulation of the fuzzy PID control strategy is carried out which provides a theoretical basis for the actual application of the flange.

\section{MATHEMATICAL MODEL OF THE FORCE CONTROL SYSTEM}

Figure 1 is the schematic of the new valve controlled air spring system. The cavity pressure, volume and initial effective area of the air spring in Figure 1 are respectively $\mathrm{P} 0, \mathrm{~V} 0$ and $\mathrm{A} 0$; the control voltage of the proportional valve is the signal $u$, and $x$ is the offset of the ends of the air spring controlled by the system.

The actual gas equation is influenced by many factors. In order to simplify the mathematical model of the force control system, we make the following assumptions[6]: The working medium is ideal gas, meeting the condition of ideal gas state equation and the law of conservation of mass; the air supply pressure $P_{s}$ and the temperature $T_{s}$ are constants; the kinetic energy and potential energy of the gas can be ignored; there is no heat exchanged between the air spring and the outside in the process action; the thermodynamic process of the gas in the air spring is quasi-static process; the leakage of the gas is neglected and so on.

Under the aforementioned condition, the air flow coming in and out should be equal to the change rate of the gas mass in the air spring. According to the law of conservation of mass, the pressure-flow formula can be expressed as follows:

$q_{m}=\frac{d m}{d t}=\frac{d}{d t}\left(\rho V_{0}\right)$

*Corresponding author: hting104@163.com

This is an Open Access article distributed under the terms of the Creative Commons Attribution License 4.0, which permits unrestricted use, distribution, and reproduction in any medium, provided the original work is properly cited. 


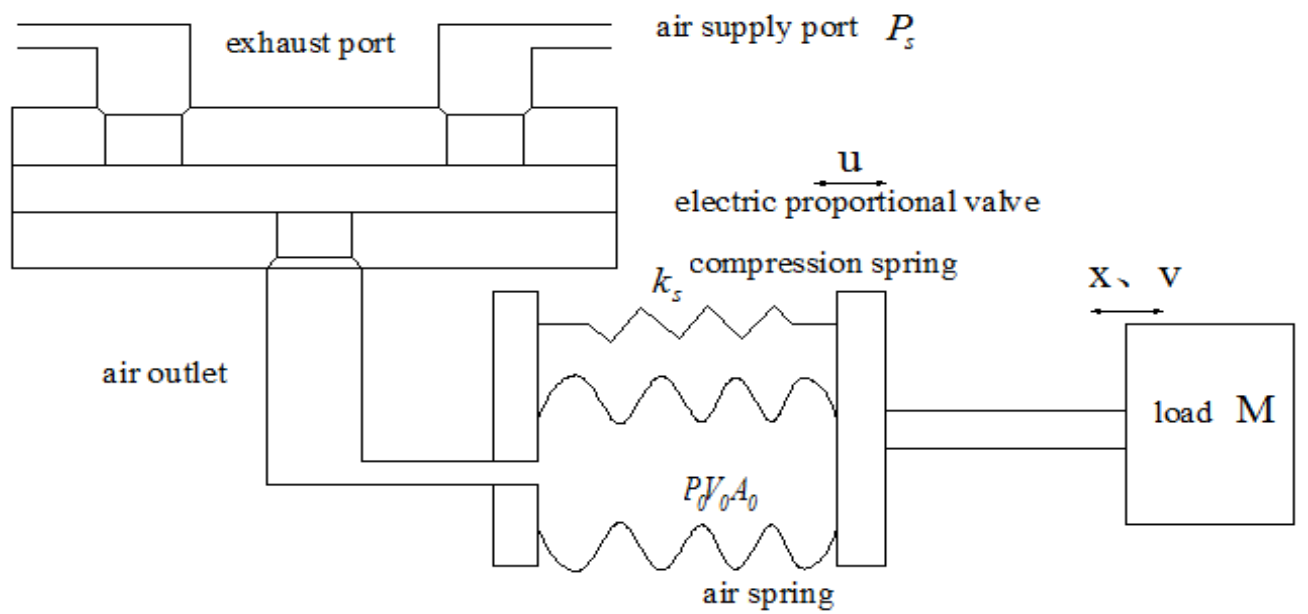

Figure 1. Valve controlled air spring system schematic diagram

Combining Equation (1) with the ideal gas state equation, and assuming the initial temperature $T_{s}$ and processing temperature $T_{0}$ meeting the adiabatic process, it is shown as follows:

$q_{m}=\frac{V_{0}}{R T_{0}}\left(\frac{P_{0}}{V_{0}} \frac{d V_{0}}{d t}+\frac{1}{k} \frac{d P_{0}}{d t}\right)$

In Equation (2), $\mathrm{R}$ is the ideal gas constant.

Equation (2) can be further modified in the condition of small disturbance as follows:

$$
\Delta q_{m}=\frac{1}{R T_{0}}\left(P_{0} A_{0} \frac{d \Delta x}{d t}+\frac{V_{0}}{k} \frac{d \Delta P_{0}}{d t}\right)
$$

According to the Sanvile flow formula, the flow of valve port has a relationship to the pressure of outlet port, the active area of valve port and the movement frequency of valve core. The movement and frequency of valve element are influenced by control voltage. Thus, we can obtain as follows:

$q_{m}=f\left(u, P_{0}\right)$

Where, $u$ is the control voltage of electric propor tional valve. Equation (4) can be linearized as follows:

$\Delta q_{m}=K_{1} \Delta u+K_{2} \Delta P_{0}$

Where, $K_{1}=\left.\frac{\partial q_{m}}{\partial \mathrm{y}}\right|_{\mathrm{y}_{0}}, K_{2}=\left.\frac{\partial q_{m}}{\partial P_{0}}\right|_{P_{0}}$.

Substitution of Equation (3) into (5), then we can obtain as follows:

$\frac{1}{R T_{0}}\left(P_{0} A_{0} \frac{d \Delta x}{d t}+\frac{V_{0}}{k} \frac{d \Delta P_{0}}{d t}\right)=K_{1} \Delta u+K_{2} \Delta P_{0}$

According to the compliant flange model as shown in Figure 1, there is an equation based on Newton's second law:
$M \frac{d^{2} x}{d t^{2}}+f \frac{d x}{d t}+F=P_{0} \cdot A_{0}-k_{s} \cdot x-F_{f}$

Equations (6) and (7) constitute the basic mathematical model of the compliant flange. Taking them into Laplace transformation, we can obtain as follows:

$\frac{1}{R T_{0}}\left(P_{0} A_{0} s \Delta X(s)+\frac{V_{0}}{k} s \Delta P_{0}(s)\right)=K_{1} \Delta U(\mathrm{~s})+K_{2} \Delta P_{0}(s)$

$\left(M s^{2}+f \cdot s+k_{s}\right) \Delta X(s)=\Delta P_{0}(s) \cdot A_{0}-\Delta F_{f}(\mathrm{~s})-\Delta F(\mathrm{~s})$

According to the Ref [7], the simplified stress transfer function of the model between displacement and the contact force can be expressed as follows:

$\frac{X(s)}{F(s)}=\frac{1 / K_{M}}{\frac{s^{2}}{\omega_{n}{ }^{2}}+2 \frac{\zeta}{\omega_{n}} s+1}$

Where $K_{M}$ is the gain of the sensor; $\omega_{n}$ is the inherent frequency of the valve controlled air spring model; $\zeta$ is the damping ratio.

In the actual condition, the offset $x$ of the ends of the air spring can be ignored due. Moreover, $\Delta F_{f}$ can be neglected. Thus, the relationship between the contact force and cavity pressure can be expressed as follows:

$\frac{F(s)}{P_{0}(s)}=\frac{K_{M} A_{0}\left(s^{2}+2 \zeta \omega_{n} \cdot s+\omega_{n}{ }^{2}\right)}{\left(K_{M}+M \omega_{n}^{2}\right) s^{2}+\left(2 \varsigma \omega_{n} K_{M}+\omega_{n}^{2} f\right) s+\left(k_{s}+K_{M}\right) \omega_{n}^{2}}$

Substitution of Equations (9), (10) and (11) into Equation (8), the transfer function between the contact force and the control voltage can be expressed as 


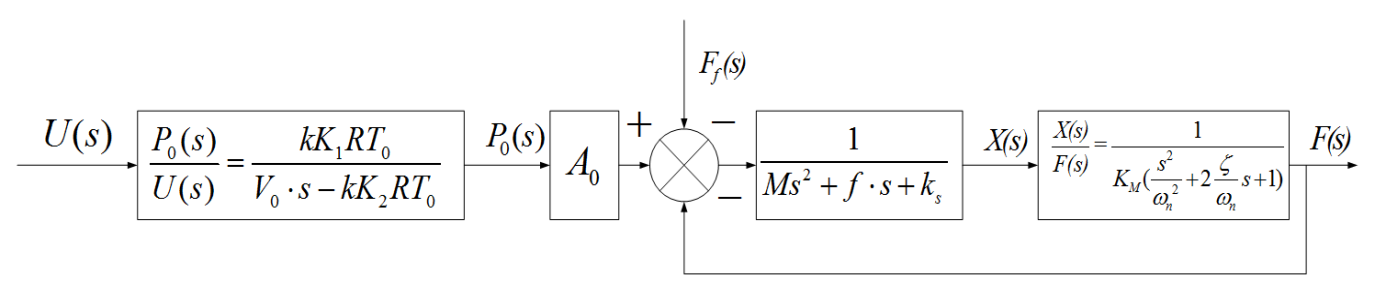

Figure 2. Schematic of the open-loop transfer function

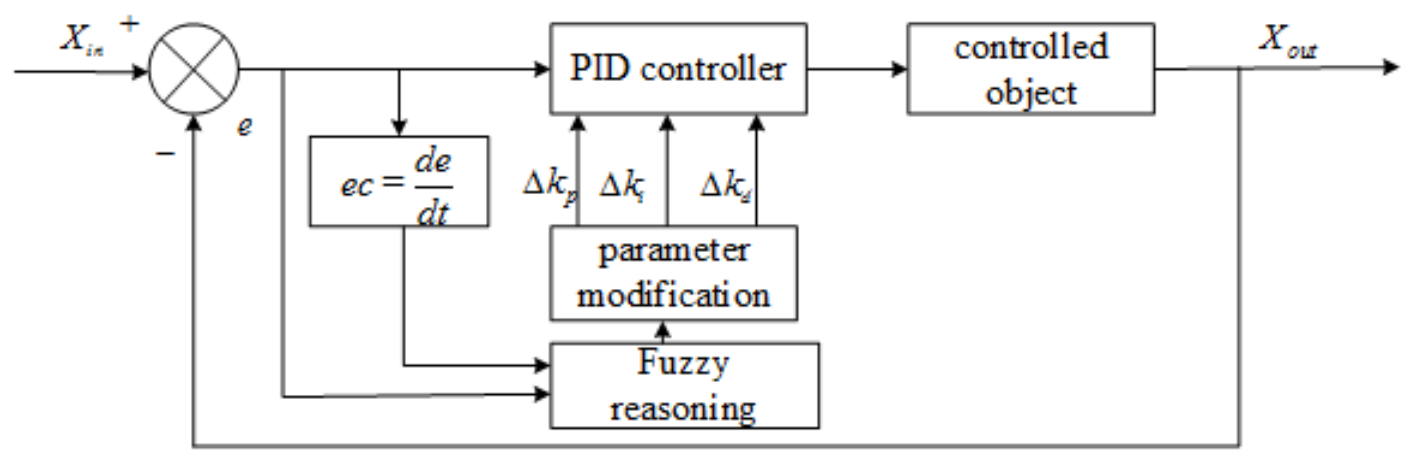

Figure 3. Self-adaptive fuzzy PID control structure

$$
\begin{aligned}
& \frac{F(s)}{U(s)}=\frac{k K_{1} K_{M} R T_{0} A_{0}\left(s^{2}+2 \varsigma \omega_{n} \cdot s+\omega_{n}^{2}\right)}{\left[\left(K_{M}+M \omega_{n}^{2}\right) s^{2}+\left(2 \varsigma \omega_{n} K_{M}+\omega_{n}^{2} f\right) s+\left(k_{s}+K_{M}\right) \omega_{n}^{2}\right]} \\
& \times \frac{1}{\left(V_{0} \cdot s-k K_{2} R T_{0}\right)}
\end{aligned}
$$

Equation (12) can be expressed by Figure 2.

Since the high-speed response of the proportional valve has little effect on the dynamic performance of the proposed system and $\mathrm{K} 2$ can be set as zero. Therefore, Equation (12) can be simplified as follows:

$$
\frac{F(s)}{U(s)}=\frac{k K_{1} K_{M} R T_{0} A_{0}\left(s^{2}+2 \varsigma \omega_{n} \cdot s+\omega_{n}^{2}\right)}{V_{0} \cdot s\left[\left(K_{M}+M \omega_{n}^{2}\right) s^{2}+\left(2 \varsigma \omega_{n} K_{M}+\omega_{n}^{2} f\right) s+\left(k_{s}+K_{M}\right) \omega_{n}^{2}\right]}
$$

According to the Equation (13), this is a third or-der control system.

\section{FUZZY PID CONTROL STRATEGY}

For PID controller, the algorithm is simple and robust. In the industrial field, PID is used in more than half of the controllers. However, the proposed grinding and polishing force control system is a typical nonlinear system, and it is difficult to establish an accurate model of the system and the traditional PID control has poor performance. The fuzzy control can imitate human's thinking process and it has good control performance on the uncertain controlled object of the mathematic model. But when the abrasive belt grinding and polishing force has little amplitude of vibra- tion at the equilibrium position, it's hard for fuzzy control to adjust the steady-state deviation of the system. In conclusion, the fuzzy PID control method was adopted by considering the advantages of both methods.

As shown in Figure 3, the system structure of the self-adaptive fuzzy PID controller was established, including the fuzzy reasoning and PID controller. The input variables are the error e and the rate of error change ec between feedback value and target value. The fuzzy reasoning is used to adjust the value of $\Delta$ $\mathrm{kp}, \Delta \mathrm{ki}$, and $\Delta \mathrm{kd}$ on line to satisfy the requirements of the different e and ec on the parameters of controller. And the controlled object will have good dynamic and static property. The control accuracy and response speed will be obviously improved.

The fuzzy subsets of input and output parameters are defined as $\{\mathrm{NB}, \mathrm{NM}, \mathrm{NS}, \mathrm{ZO}, \mathrm{PS}, \mathrm{PM}, \mathrm{PB}\}$ in the region of the fuzzy control, which the elements in the definition are respectively standing for negative large, negative medium, negative small, zero, positive small, positive medium and positive large. The theoretical area of the elements is $[-6,6]$ and those of $\Delta \mathrm{kp}, \Delta \mathrm{ki}$, and $\Delta \mathrm{kd}$ are respectively $[-0.3,0.3],[-0.3,0.3]$ and $[-3,3]$. The chosen membership functions of the input and output are triangle as shown in Figure 4.

According to the different influence of the parameters $k_{p}, k_{i}$, and $k_{d}$ on the output characteristic of the system, some rules are used to adjust parameters $k_{p}, k_{i}$ and $k_{d}$ as follows:

(1) In the condition of the beginning and stopping of the system, the error $e$ of the grinding and polishing 


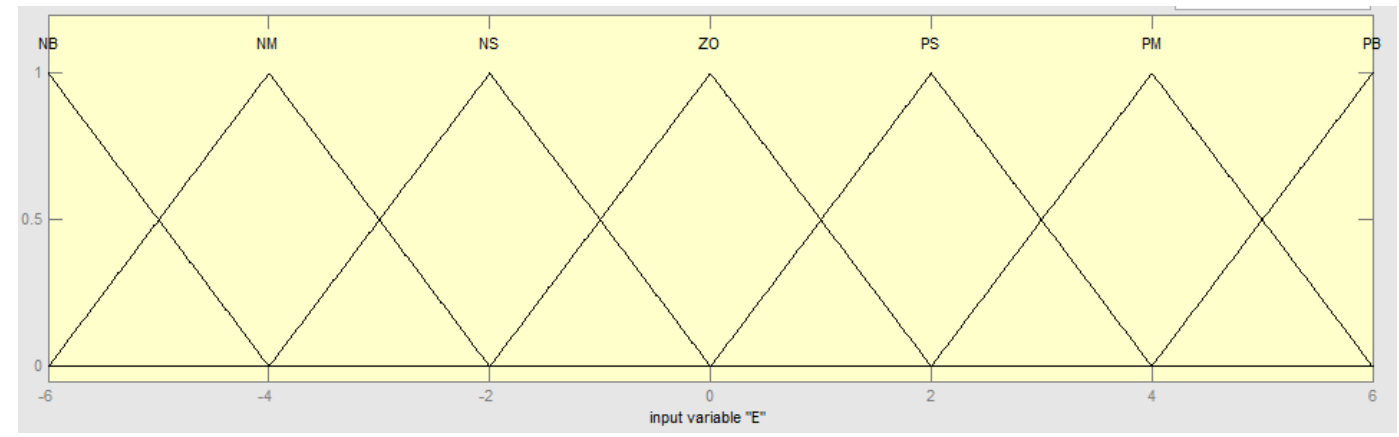

Figure 4. Membership function curve and distribution of the input parameter $\mathrm{E}$

Table 1. Fuzzy control rules

\begin{tabular}{llllllll}
\hline $\mathrm{e}$ & $\mathrm{NB}$ & $\mathrm{NM}$ & $\mathrm{NS}$ & $\mathrm{ZO}$ & $\mathrm{PS}$ & $\mathrm{PM}$ & $\mathrm{PB}$ \\
\hline $\mathrm{NB}$ & $\mathrm{PB} / \mathrm{NB} / \mathrm{PS}$ & $\mathrm{PB} / \mathrm{NB} / \mathrm{NS}$ & $\mathrm{PM} / \mathrm{NM} / \mathrm{NB}$ & $\mathrm{PM} / \mathrm{NM} / \mathrm{NB}$ & $\mathrm{PS} / \mathrm{NS} / \mathrm{NB}$ & $\mathrm{ZO} / \mathrm{ZO} / \mathrm{NM}$ & $\mathrm{ZO} / \mathrm{ZO} / \mathrm{PS}$ \\
$\mathrm{NM}$ & $\mathrm{PB} / \mathrm{NB} / \mathrm{NS}$ & $\mathrm{PB} / \mathrm{NB} / \mathrm{NS}$ & $\mathrm{PM} / \mathrm{NM} / \mathrm{NB}$ & $\mathrm{PS} / \mathrm{NS} / \mathrm{NM}$ & $\mathrm{PS} / \mathrm{NS} / \mathrm{NM}$ & $\mathrm{ZO} / \mathrm{ZO} / \mathrm{NS}$ & $\mathrm{NS} / \mathrm{ZO} / \mathrm{ZO}$ \\
$\mathrm{NS}$ & $\mathrm{PM} / \mathrm{NB} / \mathrm{ZO}$ & $\mathrm{PM} / \mathrm{NM} / \mathrm{NS}$ & $\mathrm{PM} / \mathrm{NS} / \mathrm{NM}$ & $\mathrm{PS} / \mathrm{NS} / \mathrm{NM}$ & $\mathrm{ZO} / \mathrm{ZO} / \mathrm{NS}$ & $\mathrm{NS} / \mathrm{PS} / \mathrm{NS}$ & $\mathrm{NS} / \mathrm{PS} / \mathrm{ZO}$ \\
$\mathrm{ZO}$ & $\mathrm{PM} / \mathrm{NM} / \mathrm{ZO}$ & $\mathrm{PM} / \mathrm{NM} / \mathrm{NS}$ & $\mathrm{PS} / \mathrm{NS} / \mathrm{NS}$ & $\mathrm{ZO} / \mathrm{ZO} / \mathrm{NS}$ & $\mathrm{NS} / \mathrm{PS} / \mathrm{NS}$ & $\mathrm{NM} / \mathrm{PM} / \mathrm{NS}$ & $\mathrm{NM} / \mathrm{PM} / \mathrm{ZO}$ \\
$\mathrm{PS}$ & $\mathrm{PS} / \mathrm{NM} / \mathrm{ZO}$ & $\mathrm{PS} / \mathrm{NS} / \mathrm{ZO}$ & $\mathrm{ZO} / \mathrm{ZO} / \mathrm{ZO}$ & $\mathrm{NS} / \mathrm{PS} / \mathrm{ZO}$ & $\mathrm{NS} / \mathrm{PS} / \mathrm{ZO}$ & $\mathrm{NM} / \mathrm{PM} / \mathrm{ZO}$ & $\mathrm{NM} / \mathrm{PB} / \mathrm{ZO}$ \\
$\mathrm{PM}$ & $\mathrm{PS} / \mathrm{ZO} / \mathrm{PB}$ & $\mathrm{ZO} / \mathrm{ZO} / \mathrm{NS}$ & $\mathrm{NS} / \mathrm{PS} / \mathrm{PS}$ & $\mathrm{NM} / \mathrm{PS} / \mathrm{PS}$ & $\mathrm{NM} / \mathrm{PM} / \mathrm{PS}$ & $\mathrm{NM} / \mathrm{PB} / \mathrm{PS}$ & $\mathrm{NB} / \mathrm{PB} / \mathrm{PB}$ \\
$\mathrm{PB}$ & $\mathrm{ZO} / \mathrm{ZO} / \mathrm{PB}$ & $\mathrm{ZO} / \mathrm{ZO} / \mathrm{PM}$ & $\mathrm{NM} / \mathrm{PS} / \mathrm{PM}$ & $\mathrm{NM} / \mathrm{PM} / \mathrm{PM}$ & $\mathrm{NM} / \mathrm{PM} / \mathrm{PS}$ & $\mathrm{NB} / \mathrm{PB} / \mathrm{PS}$ & $\mathrm{NB} / \mathrm{PB} / \mathrm{PB}$ \\
\hline
\end{tabular}

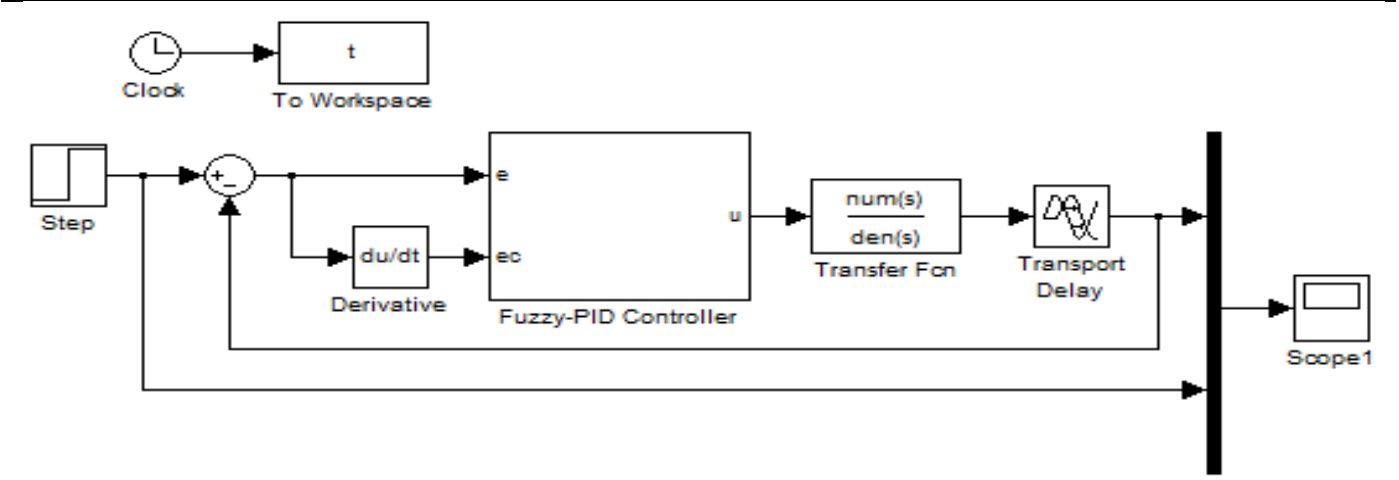

Figure 5. Simulation diagram of force control system force is larger. In order to get faster response speed, the value of $\Delta k_{p}$ should be larger; to avoid the differential supersaturation produced by the instantaneous arising of the error $e, \Delta k_{d}$ should be medium; $\Delta k_{i}$ should be zero in order to prevent the larger overshoot of the contact force.

(2) When the system is in normal operation and the error $e$, the rate of error change $e c$ in the medium range, $\Delta k_{p}$ should be small and $\Delta k_{i}$ should be suitable to ensure the smaller overshoot of the force. And $\Delta k_{d}$ should be medium because it has more effects on the response speed of the system.

(3) When the force is basically constant and the error $e$ is smaller, $\Delta k_{p}$ and $\Delta k_{i}$ should be increased. At the same time, to avoid the oscillation phenomenon near the pre-setting value of the system, and considering the anti-jamming performance, $\Delta k_{d}$ should be appropriate. Generally $\Delta k_{d}$ should be large when $e c$ is small; $\Delta k_{d}$ should be small when ec is large.

Combining the above rules, the fuzzy control table is given in Table 1 .

Fuzzy inference system uses Mamdani inference algorithm. Use the following formula to calculate the values of $k_{p}, k_{i}$ and $k_{d}$ after confirming $\Delta k_{p}, \Delta k_{i}$ and $\Delta k_{d}$

$$
\left\{\begin{array}{l}
k_{p}=k_{p}^{0}+\Delta k_{p} \\
k_{i}=k_{i}^{0}+\Delta k_{i} \\
k_{d}=k_{d}{ }^{0}+\Delta k_{d}
\end{array}\right.
$$


ICETA 2015

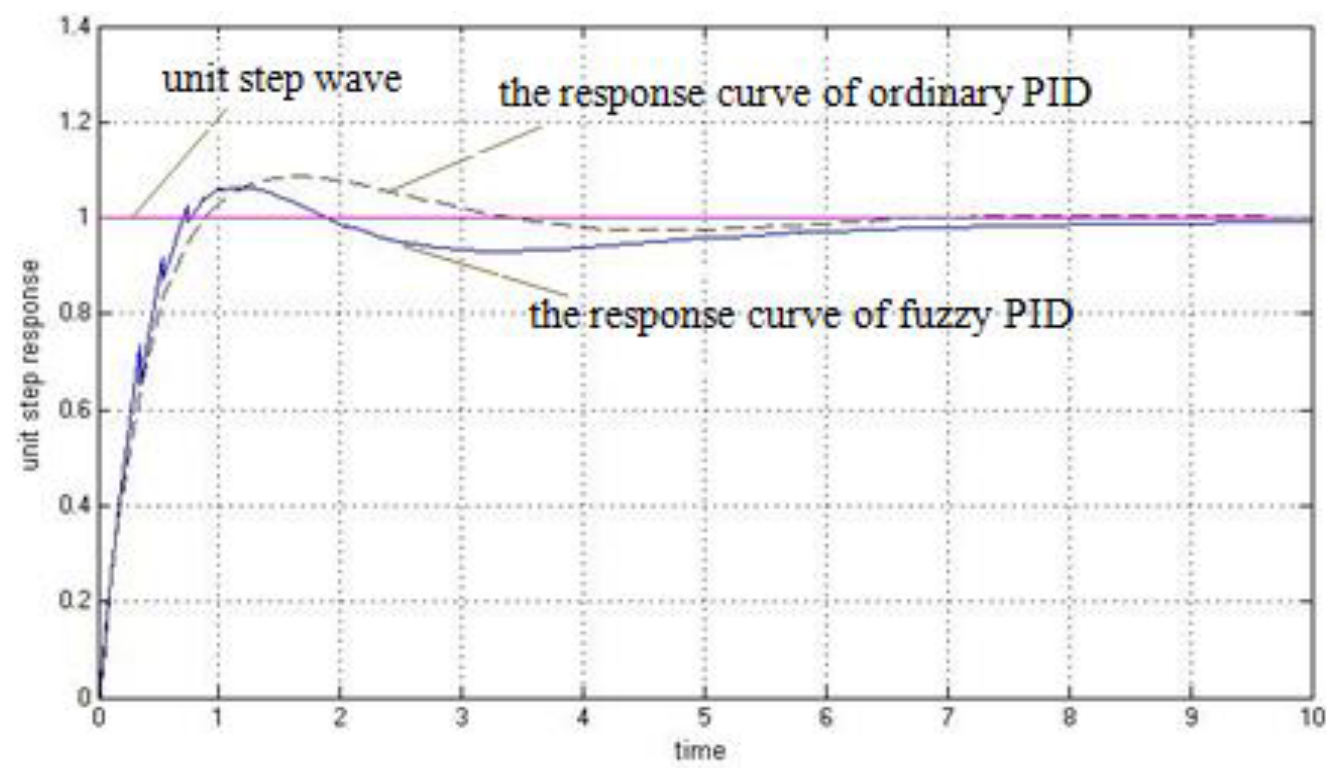

Figure 6. Output response curve of the unit step signal simulation

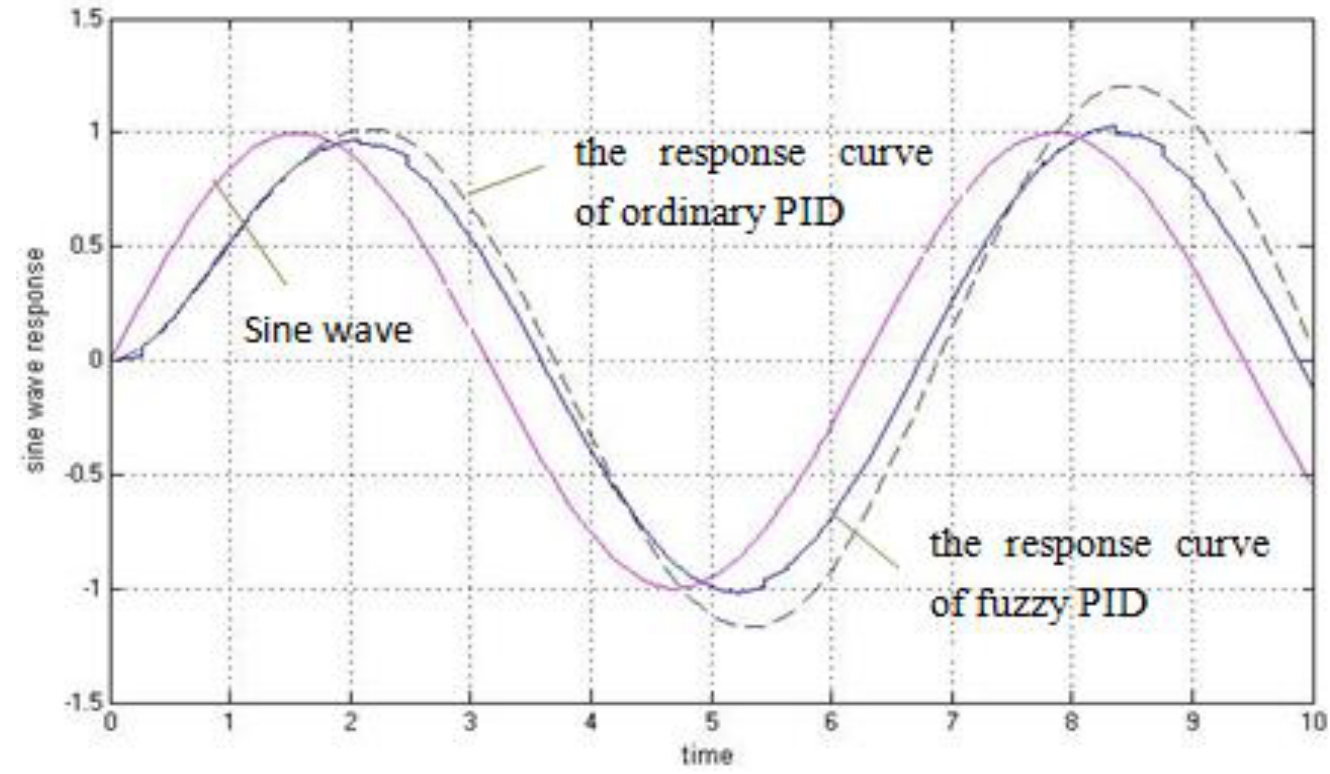

Figure 7. Output response curve of the sine signal simulation

In Equation (14), $k_{p}{ }^{0}, k_{i}^{0}$ and $k_{d}{ }^{0}$ are the pre-setting values.

The self-adaptive fuzzy PID controller established by this method submits to standard control rules and it has good versatility for the constant force control system.

\section{SIMULATION EXPERIMENTS}

In order to verify the feasibility of the scheme, the simulation was carried out by Simulink module in Matlab. The predetermined value of the PID parameters has been encapsulated into the fuzzy PID controller according to the simulation block diagram as shown in Figure5. Its working principle can be described as follows: Input the unit step signal (or sine 


\section{MATEC Web of Conferences}

signal) through a fuzzy PID controller to the established control system; compare the output with the desired value and get the error and the rate of error change; then make the result go through the fuzzy PID controller and get the control voltage parameter outputting the graphics.

A mathematical model comes out after inputting the correlation coefficient:

$$
\frac{F(s)}{U(s)}=\frac{1+0.0222 s+0.0253 s^{2}}{4.6646 e^{-5} s+9.6096 e^{-7} s^{2}+1.0439 e^{-6} s^{3}}
$$

Figure 6 and Figure 7 are the output response curve of valve controlling air spring system after choosing the appropriate gain parameters and simulating. Among them, the input signal of Figure 6 is a unit step signal and it is a sine signal for Figure 7.The solid line stands for the output response curve of the self-adaptive fuzzy PID, the dotted line stands for the response curve of ordinary PID.

After contrasting the curves from Figure 6 and Figure 7, we find that the fuzzy PID control has a faster response speed and smaller overshoot compared with the ordinary PID both for the unit step and the sine signal simulation. The value of the grinding and polishing force decides the processing quality of parts in the constant force control system. And the response speed of the contact force has an important influence on the machining quality of parts. The reduction of response time can make the force reach the pre-setting value quicker and greatly improve the performance of the constant force control system.

\section{CONCLUSIONS}

(1) This paper introduces a new compliance robotic flange actuated by the air spring. The mathematical model of the valve controlled air spring system has been established which provides a theoretical basis for the constant force control.

(2) The established mathematical model indicates that the output value of the grinding and polishing force is mainly influenced by the temperature of the gas, the effective stress area and volume of the air spring, the elastic coefficient of the compression spring, etc.

(3) The simulation results show that the fuzzy PID control has a faster response speed, a smaller overshoot and better control performance than traditional PID.

\section{ACKNOWLEDGEMENT}

This work was financially supported by the National Natural Science Foundation of China (61305118), the Natural Science Foundation of Jiangsu Province (BK20130329).

\section{REFERENCES}

[1] Yin Yuehong, Zhu Jianying. \& Wei Zhongxin. 1997. Study on hybrid kinematic analyses of force and position parallel loop for robot. Acta Aeronauticaet Astronautica Sincia, 18(6): 687-692.

[2] Zhang qing-wei, Han Lili, Xu Fang, Jia Kai. \& Zou Feng-shan. 2012. Research on hybrid position/force control for grinding robots. Control and Instruments in Chemical Industry.

[3] Erlbacher E A. 2000. Force control basis. Industrial Robot: An International Journal, 27(1): 20-29.

[4] Li Xiao-biao, Shi Yao-yao, Zhao Peng-bing. \& Duan Ji-hao. 2012. Polishing force control technology of aero-engine blade in belt polishing. Computer Inte-grated Manufacturing Systems, 18(6): 1209-1214.

[5] Duan Jihao, Shi Yaoyao, Zhang Junfeng. \& Dong Ting. 2012. Flexible Polishing Technology for Blade of Aviation Engine. Acta Aeronautica Sinica, 33(3): 573-578.

[6] Zha Hong-min. 2005. Study of pneumatic position control system based on proportional valve of direction type. Tianjin: Tianjin University.

[7] Güvenç L. \&Srinivasan K. 1995. Force controller design and evaltion for robot-assisted die and mould polishing Mechnical systems and signal processing, 9(1): 31-49. 